\title{
STANDARDS FOR THE Ph.D. DEGREE IN THE MOLECULAR BIOSCIENCES
}

\section{Recommendations of the Committee on Education of The International Union of Biochemistry and Molecular Biology}

These Standards and Recommendations are free of copyright

Printed in Canada

\section{CONTENTS}

I. Preface

II. Rationale

III. The Ph.D. Degree in the Molecular Biosciences

IV. Standards

1) Knowledge of Molecular Bioscience

2) Familiarity with the Literature

3) Recognition of Meaningful Questions

4) Technical Skills

5) Communication Skills

6) Designing Protocols and Conducting Research

V. Integrity in Science

VI. Role of Formal Graduate Courses

VII. Responsibilities of the Principal Supervisor

VIII. Responsibilities of Academics Other Than the Principal Supervisor

IX. Responsibilities of the Candidate

X. Funding of Doctoral Training

XI. Duration of Doctoral Training

XII. Doctoral Thesis

XIII. Concluding Remarks

XIV. Consultants

\section{PREFACE}

Biological Science has been changing at a stunning pace with unprecedented growth, deepening of knowledge and proliferation of methods of investigation. At the same time interdisciplinarity has become commonplace and even 
essential as the barriers between the traditional biosciences disappear. Biochemistry and molecular biology, cell biology, structural biology, developmental biology, genetics, immunology, microbiology, neurobiology, nutrition, physiology, pharmacology, and molecular medicine now speak the same scientific language and use the same molecular tools. It is not unusual for elements of these molecular biosciences to be combined in a single doctoral thesis. Besides, informational science has made possible the birth of genomics, while interest has been moving from molecules to mechanisms and to whole organisms, from a focus on individual components to biological systems.

In 1989 the Committee on Education of the International Union of Biochemistry published its Standards for the Ph.D. Degree in Biochemistry and Molecular Biology. Those Recommendations were published in: Trends in Biochemical Sciences 14, 205-209, 1989; Biochemical Education 17(2), 58-62, 1989; and FASEB Journal 3, 2106-2110, 1989. Some 3000 copies of the document and several hundred reprints were distributed throughout the world. The Recommendations were also translated into and published in Chinese and Japanese. At least one international scientific organization adapted them, and another adopted them with minor changes. Many expressed their approval and subsequently several have inquired if the Recommendations have been revised.

This document contains the revised Recommendations. The decision to revise and extend the 1989 Recommendations was not taken lightly. It was reaffirmed after a very positive response was obtained to an invitation issued to respected and experienced individuals in many countries to help the writing committee in this task. There was unanimous agreement in their responses that the 1989 Recommendations were sound and already quite generic by virtue of being expressed in behavioral terms. Because of this agreement, the format and content of the 1989 Recommendations have been largely retained so that this revision is less a matter of rewriting than of modification and updating based on the combined experience of the international and interdisciplinary panel of consultants recruited to assist in this project.

Three members of the present writing committee were heavily involved in the formulation and writing of the 1989 Recommendations. A fourth had provided comments. In this way continuity has been achieved. The majority of consultants for this revision had not previously been involved.

We thank the Executive Committee of IUBMB for their valuable assistance and financial support; the Committee on Education for entrusting us with this project; all those who acted as consultants by offering us their comments on and suggestions for revision of the 1989 Recommendations; and Dr. H. James Spooner of the College of Medicine, University of Saskatchewan, Saskatoon, Canada, for expert editorial assistance. Final responsibility for the contents of 
the present Recommendations however, rests equally with those named below.

1 October 1999

Frank Vella (Chair)

Leopoldo de Meis

Alan H. Mehler

Wilfried Rombauts

Harold B. White

Edward J. Wood

\section{RATIONALE}

During the twentieth century the preparation of students to conduct research in the Molecular Biosciences has grown from a small beginning to a major industry, producing more than 10,000 Ph.D.s per year. In the initial decades the small number of investigators who were responsible for the growth of the various fields comprised a community of individuals informed about each other's activities and aware of the status of bioscience research throughout the bioscience world. At that time instruments and techniques were relatively simple, the rate of change in the various fields was relatively slow and the judgements of the established investigators about advancing their apprentices to independence were generally similar. However, as a result of explosive growth and fragmentation into subspecialities, the thousands of scientists qualified to supervise professional training in the Molecular Biosciences now comprise a heterogeneous group, and the informal methods of the past no longer serve to maintain similar standards among nations, among institutions within a country, or even within the same institution.

Biochemistry and Molecular Biology and the related Molecular Biosciences that apply chemical, physical, and molecular biological methods and principles to the solution of biological problems (including those of biomedical and agricultural importance) are among the most vigorous and productive areas of scientific development. However, while a large number of investigators have continued to develop the intellectual and experimental aspects of these sciences, there is anecdotal evidence that Ph.D. degrees are being awarded to individuals who are poorly prepared to contribute to scientific progress or to apply science to practical problems. The profession must recognize changes in itself and its environment so as to adapt and to meet better the challenges of a fast-changing world. Although progress in scientific knowledge and understanding does not come equally from all members of the profession, most of society accepts that one Ph.D. in a particular bioscience is the equivalent of another. Although many institutions produce Ph.D. graduates of very high standard, there are bioscience departments that do not contribute significantly to the international literature yet award Ph.D. degrees. Departments in which the research capability is low should be discouraged from offering Ph.D. 
programs. Similarly, formal courses should not be a substitute for a significant research program. Differences in competence of individuals appointed as postdoctoral fellows or junior faculty members are great enough to indicate the need for international bioscience organizations to formulate acceptable standards.

Among the reasons for the differences in professional training is the diversity of educational systems in various countries and of attitudes and philosophies of institutions and individual professors. This leads to students being prepared in very different ways to enter professional study. However, the Ph.D. is recognized internationally as an award for published or publishable original research normally evaluated on the basis of a thesis. Whatever the methods used, the end result should be the same: a holder of a Ph.D. in a Molecular Bioscience should have the knowledge, skills, perspectives and understanding to be capable of self-directed scientific work of a quality satisfactory to others in the field. Although the holder of a Ph.D. is a highly qualified professional of maturity and intelligence who has acquired analytical and problem-solving skills that will eventually allow him or her to carry out independent scientific work, it is well recognized that maturation into a fullfledged independent scientist normally requires a period of post-doctoral experience that provides further refinement and specific training for the career of choice.

The experiences that have brought illustrious investigators into the biomolecular sciences have been so varied that it would be presumptuous to try to design an ideal program of education and training. Further, in fields that are still evolving rapidly, scientists looking to the future must not be fettered by restrictions imposed by others. Therefore, rather than prescribing procedures to be followed, this document emphasizes behavioral abilities that should be characteristic of those awarded a Ph.D. degree in a Molecular Bioscience, suggests how these abilities may be acquired and some methods by which progress toward attaining the abilities may be assessed, and proposes criteria for the overall evaluation of candidates for the degree. These guidelines are intended as an aid to university departments and boards of graduate studies, to national organizations that set standards for graduate education, to those scientists who serve as external examiners to evaluate theses, and to candidates for doctoral degrees in these sciences.

This revision comes at a time of strong competition in research and increased industrial sponsorship of academic research, forces that place emphasis on the rapid publication of results, on the development of practical applications, and on confidentiality. These have decreased the control over preparation of candidates at the doctoral level by academic and publicly-funded research institutions whose very existence is predicated on the production and transfer of knowledge. The Standards here defined are intended to emphasize quality in the preparation of doctoral candidates for scholarship or other productive 
careers and to de-emphasize over-specialization.

\section{THE Ph.D. DEGREE IN THE MOLECULAR BIOSCIENCES}

The purpose of a Ph.D. program is to educate and train competent, reliable, and self-directed, research scientists who have a strong sense of scientific integrity. Although the Ph.D. degree allows its holders to find employment that does not involve laboratory-based research, it implies that an individual has demonstrated an ability to pursue a research problem to a meaningful conclusion and has made a significant contribution to the advancement of basic or applied knowledge. The research experience obtained in acquiring the Ph.D. degree should assure that the awardee: understands that research involves recognition, formulation, and solving of a problem, evaluation of the significance of the solution, and presentation of the results in a considered and clear manner in writing and orally; accepts the values of scientific research; and is capable of using professional standards in all professional activities e.g., teaching, practical applications, project management or administration, relations with industrial sponsors, and research.

The education of every candidate should be sufficiently varied to give a good theoretical understanding of the major techniques in current usage and should include enough practical experience to encourage the use of novel methods as may be necessary. However, specialization of productive investigators and collaborations within ultidisciplinary research teams and between established scientists in different disciplines and institutions should be recognized as characteristic of present-day research. The importance or value of a special technique or experimental approach may be short-lived as knowledge advances, while new approaches may be more appropriate for the question being addressed. Therefore, the training of students in laboratory techniques not directly involved in their own research should not be exaggerated to the detriment of other essential skills and the Ph.D. candidate should not be trained as a technician. At a time of enormous and rapid accretion of facts and observations about Molecular Biology and other aspects of the living world and rapid changes in technique, it must be emphasized that it is the interaction between observation, experiment, and theory that is fundamental to all science.

\section{STANDARDS}

The following standards are deemed to be equally important. Their numbering is therefore arbitrary. 


\section{The candidate should demonstrate a general knowledge of physics, chemistry, biology and cell biology, biochemistry and molecular biology of the particular Molecular Bioscience, and a detailed knowledge of his or her area of research.}

The knowledge of the discipline acquired by a candidate for the Ph.D. degree should be at a professional level i.e., based on an understanding of the experimental method(s) from which some of the basic principles of the science have been derived, rather than on the conclusions that others have derived from the use of these methods. This implies extensive critical reading and analysis of some of the original publications in the particular bioscience, and of review-type papers such as are published in major review journals of the Biosciences (e.g., the Trends journals, BioEssays, Cell, the Annual Reviews series, Nature, Science, Journal of Biological Chemistry, Biochemical Journal, European Journal of Biochemistry, and FASEB Journal).

Knowledge of a particular Bioscience implies familiarity with: the structure and properties of major biomolecules; the ultrastructure of cells, organs and tissues; the major metabolic pathways; the principles of regulation of biological phenomena; cell signalling; genomics, gene expression, structure and replication; the use of international databases; and the experimental basis for some of the current canonical knowledge, paradigms, and models in the particular area of research. Professional knowledge, appreciation of the historical development of the particular Bioscience, and understanding of molecular phenomena as components of cells that function in appropriate systems integrated with others in whole organisms, should be attained by the time the candidate is awarded the Ph.D. degree. Because the extent to which such knowledge is acquired during under-graduate education varies, it is important to assess the candidate's knowledge early enough so that supplementary education during the Ph.D. training period may be adjusted to the need.

Attainment of appropriate knowledge and insights may be evaluated formally (by essay writing, comprehensive oral tests, periodic written reports on specific seminars the candidate has attended, a written literature review defended orally, etc.) or informally (by questioning on matters relating to the research proposal, during discussions on progress, seminars or journal-club presentations made by the candidate, and review of early drafts of the candidate's thesis). Although ongoing or formative evaluation is likely to be mainly the supervisor's responsibility, the responsibility for periodic cumulative evaluation should be shared with the supervisory committee.

The far-reaching developments in informatics, universalization of coverage by the Internet, and increasing availability of access to scientific literature via this medium require that Internet information retrieval resources and formal courses in Bioinformatics should be available to doctoral candidates. While the skills 
necessary for use of information technology (for example computer literacy, data processing, database searching, use of Internet, and CD-ROM databases) may soon become an integral part of secondary and undergraduate education, doctoral programs should ensure that candidates acquire and exercise these skills.

It is impossible to function in modern bioscience research without a working knowledge of English. Over $90 \%$ of the published literature is in English. This is also the language of international congresses and of the Internet. In many countries candidates are required to demonstrate competence in speaking and writing of this language, for example by satisfactory completion of the TOEFL test or an equivalent. Where English is not the first language, candidates can be helped by the use of English in a significant part of their education and training. The presentation of seminars, papers at journal-club meetings, and written reports in English are good ways of fostering confidence in this language of modern science.

\section{The candidate should be familiar with the research literature of the particular Bioscience and should have the ability to keep abreast of major developments and to acquire a working background in any area.}

To define and formulate the thesis problem and possible ways to solve it require skill in information retrieval and information processing. This implies familiarity with the literature of the particular Bioscience that contains not only the results of investigations conducted by established scientists, but also their reasoning, experimental strategies, descriptions of technique and materials, discussion of results and evaluation of hypotheses, and the models of processes and phenomena that summarize much of the accumulated wisdom of the discipline. Furthermore, familiarity with the literature identifies areas that have already been explored or which require exploration and those where available results or interpretations are still controversial. The literature is the major link between bioscientists throughout the world and is the repository of a vast and increasing amount of scientific information. Candidates should contribute to this literature during their training and possibly throughout their career. The abilities to access and review the literature, to evaluate it critically, to abstract from it the useful and the valid as a basis for further exploration or investigation, are essential skills for a self-directed bioscientist.

While it is important for candidates to obtain and evaluate data, they must also comprehend what the data represent, develop the capacity to use and extend the knowledge generated, appreciate the significance of their original contributions to knowledge, and acquire the skills necessary for effective oral and written communication. Avenues for the development and evaluation of these abilities include: preparing of proposals for research and for grants, scanning several journals regularly to maintain a general view of the Molecular Biosciences, making seminar and journal club-type presentations in the area of 
the thesis as well as outside it, preparing results for publication, periodically reviewing progress, and preparing the thesis. Some involvement in undergraduate teaching (e.g., as a teaching assistant, tutor, or laboratory demonstrator) helps to encourage and sustain a breadth of interest beyond the thesis topic, to develop instructional skills, and to consider teaching as a career option.

\section{The candidate should demonstrate skill in the recognition of meaningful problems and questions for research in the particular Bioscience.}

This ability arises in part from familiarity with and critical evaluation of the general literature of the particular Molecular Bioscience. It requires broad and detailed knowledge, creativity, and imagination, and is facilitated by discussion with other scientists. Meaningful problems and questions raised by them are circumscribed and solvable by rigorous experimentation, of interest to others working in related areas, and often require the acquisition of new technical skills. Their answers become part of accepted scientific knowledge and contribute to the basis for further research.

The ability to evaluate questions and to define attainable objectives is developed by responding to questions raised by the supervisor within an educational setting, analysis of questions asked in published papers or scientific seminars, the raising of questions on the basis of results in specific papers and seminars, the drafting and defence of research proposals, periodic review of the doctoral research, and preparation of the thesis. Research dedicated mainly to acquisition of technical experience should be discouraged as thesis work.

The candidate should have access to structured experiences the major objectives of which are to provide opportunities to present and defend research plans and their results and interpretations, to evaluate and comment on the work of others, and to participate in discussions about technical and scientific issues. Active participation in research seminars and attendance at regional, national, and international meetings should be encouraged so that candidates can establish networks, and engage in scientific discussion, expand their horizons, and acquire the skills necessary for collaboration with other scientists.

Acquisition of the ability to recognize meaningful questions and to formulate testable hypotheses with appropriate controls is a major step in the candidate's transition from a passive to an active role in Bioscience research. Attendance at and scheduled contributions to regular laboratory group meetings foster this ability. One way of evaluating this skill is to require a candidate to make one or more oral presentations after a brief preparation time on a topic or topics unrelated to that of the thesis. The candidate could be required also to identify questions from these topics that deserve further study, to identify further and choose between multiple pathways of knowledge discovery, to present and 
discuss possible experimental approaches that might be used to obtain answers to such questions, and to write the protocols appropriate for such experimental approaches.

\section{The candidate should possess technical skill in laboratory manipulation.}

When candidates have received little training in laboratory technique, as frequently happens when their only background is that of an undergraduate degree, it is good practice to separate the first year for laboratory rotations and development of transferable skills from the period dedicated mainly to the doctoral research. The skill of keeping detailed and accurate records of experimental work in appropriately indexed laboratory notebooks should be acquired at this stage. Departments and institutes should resist the tendency to overemphasize the training element (e.g. the learning of as many techniques as possible, the development of technical competence) that reduces a doctoral program to a technician-training vehicle and has little regard for educational elements or the scientific outcome of publishable results.

Because the number of experimental techniques is very large, the doctoral candidate cannot acquire formal training in every available technology. Rather, the candidate should acquire enough skill in the basic techniques of biochemistry and molecular biology and sufficient technical competence to be able to function competently and efficiently. This includes the ability to devise and perform the experiments required to solve the problem and to evaluate critically the information generated. The candidate should demonstrate capability in the laboratory techniques related to the research project, the principles on which the apparatus used are constructed, a good understanding of quality control in the laboratory, the theoretical basis for these techniques, and sufficient self-confidence and competence in laboratory methodology so as not to be inhibited in adopting novel technology as may be required for undertaking research in the future. Technical competence and flexibility are essential tools for self-directed research. While the use of commercial kits has become commonplace, when these are employed in the research work, candidates should have a clear understanding of the theoretical basis, their components, and their advantages and disadvantages.

Part of a candidate's experience should include locating, pricing, and ordering of equipment and consumables associated with the research. These activities require familiarity with catalogues, a habit of reading product brochures provided by commercial suppliers, visiting product exhibitions at meetings, and familiarity with shopping via the Internet. Avenues for development of this ability include the carrying out of the experimentation for the thesis, specially designed laboratory courses or workshops, or short periods of training in other laboratories. 
The research infrastructure available to doctoral candidates in different countries, and even in different universities within a country, are highly variable. One solution to this problem is through inter-institutional cooperation and programs that permit candidates to spend part of their training period outside their home institutions in order to attend specialized laboratory courses or workshops or to participate in collaborative research. This avenue is more likely to be available where supervisors have well-established and effective professional networks.

Candidates should be aware of and expected to adhere to current guidelines concerning human experimentation and the use of animals in research, laboratory safety, and the use of recombinant DNA technology including transgenic species.

\section{The candidate should demonstrate that oral, written, and visual communication skills have been acquired.}

The value of scientific research depends on the effective communication of results and their interpretation to the scientific community. Scientists communicate by giving lectures and seminars, designing attractive and informative posters, writing publishable manuscripts, applying for grant support, and by speaking to non-scientists. Communication skills in science place a premium on logical argumentation and clarity in speech and writing. Candidates learn these skills through practice and develop confidence over time. While writing and oral presentations should be part of graduate courses, the entire graduate education should emphasize and integrate communication skills. These are largely generic and prove useful should the Ph.D. graduate opt for career paths other than research.

There are many opportunities during the doctoral process for developing communication skills: e.g., in the preparation of the research proposal, the periodic review of research progress, the preparation and oral defense of the thesis, preparation of research material for publication, journal-club presentations and seminars on and outside the thesis topic, preparation of grant proposals based on library projects, and oral and poster presentations at scientific societies or national meetings. An important aspect of such activities is the critical review of presentations made by the candidate. Opportunities should also be taken for discussion of ethical aspects in the presentation of results, consultation with all co-authors, and attribution of credit for the work and materials of others, including appropriate delineation of their role in any publication.

It is the responsibility of the principal supervisor and supervisory committee, and of the department or institute where the candidate is to work, to indicate to 
the candidate at the beginning of doctoral training what is expected, what the standards are, and to provide positive feedback and guidance at every opportunity.

\section{The candidate should demonstrate skill in designing experimental protocols and in conducting productive self-directed research.}

These skills are of fundamental importance for self-directed research in the Biosciences. Their acquisition is demonstrated by the successful completion of a self-initiated piece of research that leads to publication in an international peer-review journal. This involves the asking of questions at an appropriate level (not too trivial, not too large), the carrying out of appropriate and reproducible experiments with suitable controls and good quality-assurance, treating data statistically and analyzing the results, deriving of answers to the questions posed, and their acceptance by the scientific community by refereed publication. Also important is the development of testable models to explain experimental results and increase understanding of molecular topics of the research.

This skill is not acquired simply by the collection or compilation of data, by cataloguing of observations, or by other activities in which the candidate serves as a technician. The candidate must participate actively in the selection of the problem. Supervisors should assist in orienting their candidates to the relevant literature. The supervisor and the supervisory committee should participate in periodic evaluation of the progress in a critical way but should permit the student to carry out independently planned experiments and even to learn from mistakes (within reasonable limits set by budgetary and safety considerations). Thus, relatively inexpensive and short experiments are allowable but expensive and relatively long ones need to be planned very carefully. Candidates should be encouraged to estimate the risks involved in carrying out experiments and balance these with the need to obtain data. They also need to learn the extent to which commercial reagents and materials obtained from other laboratories can be relied upon. A critical approach to all aspects of the work and the fundamental need for appropriate controls for all experiments are essential components of good science.

The original description of the thesis problem should not be too restrictive. The candidate should be encouraged to recognize promising leads suggested by results, to propose experiments based on results, and be permitted to change the problem if the change appears likely to produce more valuable results. The balance between persistence in overcoming difficulties, seeking alternative procedures to reach the same goal, and wasting time on poor ideas can only be learned by experience. Similarly, the lure of tempting new ideas may have to be resisted to the extent needed to bring projects to publishable conclusions within the time constraints set, for example, by tenure of scholarships and governmental or institutional regulations. 
A written scientific report, followed by oral presentation and defence at the end of the first year (of three) or second year (of four), should be used to determine whether the research is likely to be productive enough to lead to a doctoral thesis and assess whether the candidate has the intellectual and technical abilities to succeed and the willingness to do the necessary work.

\section{INTEGRITY IN SCIENCE}

Science depends upon integrity. Results that are published in scientific papers or presented at meetings should represent honest accounts of the work done. Two major traditional functions of editors of scientific journals have been to provide an independent peer-review system for articles submitted for publication, and to eliminate inaccurate and imprecise statements before publication, so that other investigators can repeat published experiments without difficulty. Recent years, however, have seen several high-profile cases of retraction of articles because of compelling evidence on the part of one or more co-authors that the articles contained fabricated data.

The small number of well-publicized examples of publications that contain false results are evidence of the effectiveness of the self-correcting mechanisms of the scientific system. Every instance of dishonesty, no matter how trivial it may seem, has the potential to be very harmful to individual scientists and to the relationship between science and the rest of society. Because of this, all students must be educated and trained in an atmosphere of unquestioned integrity and any act of plagiarism, deliberate distortion, misrepresentation, or misleading ascription of authorship should be considered by the appropriate administrative authorities as grounds for dismissal or a severe warning with monitoring to ensure compliance with ethical standards. It is to be assumed that every department or laboratory engaged in the pursuit of scientific knowledge is characterized by an atmosphere of mutual trust, fairness, scientific honesty, and openness. Nevertheless, institutions should have procedures in place to deal with the rare occasions when unethical behavior is detected.

As the Molecular Biosciences develop even faster and as the potential for material rewards increases, competition for priority in publication becomes keener. This can lead to misrepresentation of data, fabrication or falsification of results, lack of consultation with all co-authors, and the omission of reference to related or similar published work by others. It can also lead to release of data and conclusions in the popular media prior to publication in a peer-review journal. Science remains, nevertheless, a collaborative effort and graduate education must emphasize the interdependence of scientists and the feeling of 
participation in the work of an international community of trustworthy scholars. It is assumed that those who wish to join this community accept the ethical precepts that have characterized science, and that their education will include appropriate discussion of these precepts and develop their ability to work ethically in groups. Candidates should be aware of the ethical implications of their research and of their responsibilities as scientists. Although seminars and courses are frequently seen as a way to achieve awareness of ethical standards, it is the example of role models and especially the candidate's principal supervisor that is most important. The principal supervisor should make appropriate opportunities for presenting the ethical view of science.

\section{ROLE OF FORMAL GRADUATE COURSES}

Short courses or workshops in transferable skills (e.g. on scientific writing, presentation of talks, bioethics and professional ethics, time and project management, information storage and retrieval, recording of experimental protocols and results, intellectual property rights, acquisition and management of research grants, laboratory safety, guidelines on human experimentation and handling of animals, library and computer skills, statistics) are especially appropriate in the early part of a doctoral program. They are likely to improve effectiveness and research performance and enhance the likelihood that candidates successfully enter their future careers. Such courses or workshops are frequently offered by colleges or boards of graduate studies, and by staff development units, but many large departments with many doctoral candidates organize their own. Generic or specialized courses, inter-departmental teaching, and formal student-run seminars are useful in building confidence and promoting a sense of collegiality.

Formal courses are a convenient route to the acquisition of information in a field of study. They are frequently used to expand the general information base of students. Since the primary goal of graduate education and training is the acquisition of self-direction and familiarity with the pertinent literature, formal courses are useful to the graduate program only if they prepare the candidate for life-long learning or research activity. Graduate-level courses should therefore involve the use of the literature by traditional and electronic means and be concerned with active self-education. Since independent scientists need to keep up with developments in their field, any required specialized graduate courses should be directed toward this future need.

Specialized graduate courses in the Molecular Biosciences should be designed not just to increase the knowledge base of candidates, but also to make them more professional in their work and enable them to become effective communicators. They should be interactive in style rather than in the form of traditional undergraduate lecture courses, so as to develop higher-level 
intellectual skills rather than the transient accumulation of memory-based information and the assessment procedures should test for these skills rather than for rote learning. They should also contribute to the development of a professional attitude and value system. Departments or institutions that lack adequate facilities for research at a doctoral level and in which little research is in progress should not use formal courses as a substitute for original laboratory or theoretical research. Regardless of course content or format, accumulation of credits by "passing" courses does not provide evidence that the candidate is better prepared to contribute to science. Because the Ph.D. is a research degree, grades obtained in such courses should not contribute in a major way in the final evaluation of candidates. In countries where academic and scientific resources are limited, inter-institutional cooperation can make up for local deficiencies. We consider realistic and courageous the decision of various departments of Molecular Biosciences not to offer Ph.D. programs because they lack the proper human, economic, physical, and technical resources.

It must be appreciated that courses may be time consuming and can be disruptive of experimental work, and that the knowledge and skills that they may foster can be acquired in other ways (e.g. journal-club activities, reviews of the literature on selected topics, seminars on topics unrelated to the research). Because of these considerations formal courses should be limited in number and duration and should be so selected as to permit a candidate to switch disciplines between joining a graduate program and beginning the research that will form the basis of the thesis.

\section{RESPONSIBILITIES OF THE PRINCIPAL SUPERVISOR}

A principal supervisor should have an ongoing research project and should have contributed to the peer-review literature. Supervisors, through interaction with their candidates in planning and programming of the work and in setting and keeping of deadlines, represent the most important external influence in the learning and development that occurs in doctoral candidates. Progress in doctoral research depends on the nature, frequency and quality of supervision (particularly through the giving of critical feedback and checking on progression of the work) provided to candidates, especially in the early stages. It is good and recommended supervisory practice that short notes be filed for a minimum number of meetings as agreed to by the candidate and principal supervisor.

The role of the principal supervisor in directing student research is one that requires subtle adjustments in personal perspectives and behavior towards the candidate. In general, candidates begin with little relevant knowledge, restricted skills and limited perspective, and require a considerable amount of guidance. However, the naive beginner must evolve into a self-reliant and professional investigator during the thesis work. Development of the many personal and 
professional skills necessary for research, for future careers, and for the capacity of self-direction are acquired only through practice and feedback. The supervisor must decrease detailed direction as the project proceeds and the candidate becomes more self-reliant, and may have to accept a loss in efficiency in the work of the laboratory as part of the cost of professional education. The supervisor and candidate thus gradually become mutuallyrespecting colleagues participating in a joint research project. The number of doctoral candidates that any principal supervisor can handle should be restricted to within reasonable limits.

The principal supervisor and the candidate participate as partners in a mutual effort but not as equals. This makes it essential that in the event of difficulties in the relationship, a clear and explicit route for their resolution be available. Because the process of doctoral education and training contains a major element of apprenticeship in research, the supervisor is not only a teacher and mentor but also a major determinant of the relationship of the candidate to the scientific community and of his or her subsequent professional opportunities. Creation of professional networks by a candidate (e.g. through participation in scientific meetings, through the internet) should be mediated and encouraged by the supervisor.

Because this may be the single most important career decision that a candidate makes, there should be ample time in which to choose the thesis topic and supervisor or mentor after having been exposed to several possible supervisors. Attempts by potential mentors to induce candidates to train with them other than by open methods are to be discouraged.

\section{RESPONSIBILITIES OF ACADEMICS OTHER THAN THE PRINCIPAL SUPERVISOR}

Though the doctoral education process is often viewed as being based largely on the human and scientific aspects of the supervisor-candidate relationship, the complete training of the candidate to meet these standards may be, and very frequently is, beyond the ability of one person. It must be recognized that other academics with experience in research and supervision and in specialized fields (e.g., statistics, new techniques) have an important role to play in a candidate's training and should be members of the candidate's supervisory committee. This committee should not be chaired by the principal supervisor, and should meet at least once a year, keep written records (copied to the candidate) on progress and on advice given, and include a member from outside the candidate's department. This not only broadens the scope of the learning environment for the candidate but also demonstrates the social and interactive nature of scientific research and thinking, which are practised within the local and the international scientific community and are increasingly 
dependent upon networks and a team-based approach.

Among the functions of a candidate's supervisory committee are: approval of the program of training and of the project; monitoring and periodic assessment of progress towards completion of the thesis work; decisions on the adequacy of the candidate for continuation in a doctoral program; and determination of when enough work has been done to satisfy the requirements of a thesis.

It is the responsibility of the department or institute in which the candidate is being educated and trained: to define the procedures for selection and evaluation of candidates and requirements (including timing, methods of evaluation and expected standards) for granting the degree; to provide the appropriate physical and intellectual environment in which the skills and competencies outlined in Section IV can be acquired; to ensure appropriate and proper supervision for the candidate; and to enunciate a clear policy on authorship, intellectual property, and procedures for grievance and appeal. It is also the responsibility of the institution to make provision for the development of supervisors, for guidance and tuition of candidates in the English language as may be necessary, and for providing an environment that promotes the general and professional well-being and development of its doctoral candidates.

\section{RESPONSIBILITIES OF THE CANDIDATE}

A doctoral program must be as concerned with the intellectual and scientific growth of the candidate as with the quality and merit of the work for, and reported in, the thesis. For both to be achieved satisfactorily and effectively, the candidate must be knowledgeable about and actively involved in the process and mindful of his or her responsibilities. These include: familiarity with and observance of the regulations, requirements and guidelines prepared by the institution, department, and principal and other supervisors that relate to the doctoral program and degree; familiarity with the handling and care of equipment and materials to be used in the research work; maintenance of professional and ethical relationships at all times with his or her supervisors, department, and institution; participation in and contribution to the intellectual and scientific community provided by the department and institution; attendance at all assigned courses and other activities as required by the supervisor(s), department and institution; and assurance that all original data on the research work are recorded diligently and assigned for safekeeping in the department for the period designated by the department and/or institution (this is usually no less than five years after completion of the thesis work).

A satisfactory relationship between supervisor(s) and candidate is one that is beneficial and supportive and that contributes to the shaping of attitudes, skills 
and insights of both.

\section{FUNDING OF DOCTORAL TRAINING}

In many cases the rapid expansion of doctoral training during the past fifty years has been associated with large increases in cost. In the sciences the cost has generally been provided by government funding. Recently, additional support has been received from commercial firms. In some instances, the candidate may be required to meet some of the expense. However, the source of funding should make no difference to the requirements for the awarding of the Ph.D. degree. Regardless of the source of funding, candidates for the Ph.D. degree should satisfy the same requirements and meet the same standards.

Funding by industry has been beneficial for both the universities (e.g., through their being perceived as co-operating with industry) and for the doctoral candidates concerned (e.g., through stipends that are more generous than those provided by public funding, and through better prospects for future careers). Industry also benefits through getting some of its research done on a contractual basis, enhancement of its scientific respectability, and perceived generous co-operation with universities, through having research data at its disposal soon after they are obtained, and through increased publicity.

Very frequently, however, industry insists on the signing of confidentiality agreements by the candidate, supervisor(s) and external examiner(s). This usually involves restrictions on the release of data derived from the research, be it in seminars, abstracts, posters, publications, or the thesis. It should be emphasized that the award of the Ph.D. degree should not be made on the basis of work whose validity depends upon confidential research. It is essential that research projects sponsored by industrial grants or contracts should not impose unreasonable restrictions on dissemination and publication of work done as part of a doctoral thesis, even when the data may be unfavorable to the sponsor.

\section{DURATION OF DOCTORAL TRAINING}

Many students enter graduate school or programs directly from an undergraduate degree with little preparedness for the marked differences between under-graduate and graduate science, the day-to-day uncertainty of research work, and little preparation for their experiences as graduate students or the expectations that supervisors may have of them. Transition involves many changes in status, style of work, scope of intellectual problems to be faced, 
confidence and even self-esteem. The majority of students would benefit from a year of pre-graduate studies that includes placements in laboratories of different supervisors to permit exploration of a variety of laboratory techniques and research problems, and a variety of short courses or workshops on transferable skills. At this stage, a candidate's aptitude for research and scholarship at the standard appropriate for a doctoral degree can be assessed and the option of a Master's degree can be offered to those deemed unsuitable for doctoral work.

The transition from student to self-directed scientist does not proceed at the same rate for different individuals. An even greater variable is the period for completion of research projects. It is not reasonable to expect that the requirements for a Ph.D. degree can be completed within a short period of time. Where outside forces (usually government ones) apply economic influences to restrict the time for graduate training, members of the profession should resist these pressures to award degrees prematurely or to reject students who could become useful professionals given longer periods of training. The awarding of a $\mathrm{Ph}$.D. degrees should identify an individual who has acquired high standards of scientific research and who does not compromise those standards to meet arbitrary deadlines. Since the candidate is expected to acquire or develop a professional philosophy and professional values in addition to technical knowledge and skills, regardless of success in research, it would seem reasonable that the period of training should not be less than three years with a maximum of five for full-time candidates.

The progress of every candidate should be monitored and documented by the supervisory committee. Decisions about abandoning unproductive projects should not come suddenly after several years, but should arise from discussions with the candidate while there is still time to complete the degree within the conventional period. Serious questions must be asked, and decisions made, early in the training process about the abilities of the candidate to complete the type of work which will lead to a satisfactory thesis within a reasonable time. Time limits should be flexible and retention of competent candidates within a program simply because they are productive should be discouraged.

\section{DOCTORAL THESIS}

The doctoral thesis, presented and defended orally before at least one external expert and the supervisory committee, is the ultimate evidence that the doctoral candidate has acquired the skills and abilities required for certification as a competent, self-reliant scientist. It must serve to demonstrate that the candidate has conducted successful and meaningful research by solving an original problem with an increasing degree of independence, proposing ideas of his or her own, that the candidate's contributions have been significant, and that 
the candidate understands how the results fit into the scheme of current knowledge. The writing of the thesis should be the candidate's responsibility. It should preferably be in English, or at least contain an extended summary in that language. Final evaluation of the Ph.D. thesis should be the responsibility of one or more experts invited from outside the institution. Institutional structures for review of comments of external examiners on the competence and performance of candidates contribute to ensuring uniformity in the application of standards.

The doctoral thesis may take different forms. At one extreme, it may be a lengthy document giving a thorough review of the literature, an explanation of the problem(s) selected, detailed descriptions of the methods, a complete presentation of experimental results, and an extended discussion of the interpretation and implication of the findings. At the other extreme, but one that is not universally accepted, it may consist of one or more published papers with a general introduction and a thorough discussion of the research project. Since it is not possible easily to evaluate the candidate's contribution to any formal publications, especially when there are other authors, and since journals restrict the amount of explanatory and interpretive material, the thesis should include material written by the candidate in order to supply the information beyond that included in the published papers. It should show clearly what the candidate's contribution is and how the candidate has put the research into scientific perspective. Such material should introduce each publication used as part of the thesis and should discuss the significance of the research and its implications for future investigations or applications.

Much research today is done in large laboratories in which several candidates, technicians, and post-doctoral fellows contribute to a project. Under such circumstances, clear delineation of the contribution made by the candidate is essential in a thesis, and the work done by others should be explicitly defined and acknowledged appropriately.

The size or volume of thesis material should not be used as a criterion in its evaluation. To be borne in mind when publications form part of a thesis is the high cost of publication in prestigious journals.

Prior publication of material to be included in a thesis should be encouraged. The rapid pace of scientific development requires that all meaningful and original research be published as rapidly as possible. The Ph.D. degree should normally only be awarded for a thesis which contains original work which has already been published with the candidate as first author or which is deemed by the examining body to be suitable for publication in an established, refereed journal in the field. However, it must be recognized that a candidate may sometimes meet all the requirements but not achieve results that are publishable. 
The granting of a Ph.D. solely on the presentation of published papers, without any component of formal education and training, is to be discouraged.

Award of the doctoral degree should be based solely on the demonstrated capacity of the candidate to meet these Standards.

\section{CONCLUDING REMARKS}

These Recommendations articulate the process and understanding of many of the problems involved in the education and training of self-directed scientists as signified by the award of the Ph.D. degree. Experience in various institutions and countries has shown that successful molecular bioscientists can be produced by diverse routes and systems and meet the Standards described here. It is hoped that the Recommendations and Standards here presented will prove helpful to all involved in the award of the doctoral degree in the Molecular Biosciences.

Copies of this booklet can be obtained from:

Dr. F. Vella

18 Leyden Crescent

Saskatoon, SK

Canada S7J 2S4

Fax: +1-306-955-1314

E-mail: $\underline{\text { f.vella@sk.sympatico.ca }}$

\section{ACKNOWLEDGEMENTS}

We are grateful to the following who acted as consultants:

Ishola Adamson (Abeokuta, Nigeria); Knut-Jan Andersen (Bergen, Norway); Trevor R. Anderson (Scottsville, South Africa); M. Farid El Asmar (Cairo, Egypt); G. Balakrish Nair (Calcutta, India); Richard J. Balment (Manchester, UK); Rena Balzan (Msida, Malta); Joe V. Bannister (Msida, Malta); William Bannister (Msida, Malta); Paolo Bernardi (Padova, Italy); Alfons Billiau (Leuven, Belgium); Debi P. Burma (Varanasi, India); Jeremy H. Brock (Glasgow, Scotland); Peter N. Campbell (London, UK); Juan Jose Cazzulo (Buenos Aires, Argentina); Maharani Chakravorty (Varanasi, India); D. Chattopadhyay (Calcutta, India); Roberta F. Colman (Newark, U.S.A.); Lourdes J. Cruz (Diliman, Philippines); Leopoldo de Meis (Rio de Janeiro, Brazil); Rodney Devenish (Clayton, Australia); Peter J. Dolphin (Halifax, Canada); Khoo Hoon 
Eng (Singapore); Alessandro Finazzi Agró (Rome, Italy); John B.C. Findlay (Leeds, UK); Carlos Gancedo (Madrid, Spain); Hagai Ginsburg (Jerusalem, Israel); André Goffeau (Louvain-la-neuve, Belgium); B.C. Guha (Calcutta, India); Joan J. Guinovart (Barcelona, Spain); Julia A. Hasler (Harare, Zimbabwe); Cecilia Hidalgo (Santiago, Chile); Robert L. Hill (Durham, U.S.A.); Giuseppe Inesi (Baltimore, U.S.A.); Steven G. Hillier (Edinburgh, Scotland); Ivan G. Ivanov (Sofia, Bulgaria); Yasuo Kagawa (Tochigi-ken, Japan); Raj Kalaria (Newcastle upon Tyne, UK); Manuel Krauskopf (Santiago, Chile); Tamotsu Kondow (Ichikawa, Japan); Dimitris Kyriakidis (Thessaloniki, Greece); William K. Latshaw (Saskatoon, Canada); Peter N. Lewis (Toronto, Canada); Qi-Shui Lin (Shanghai, P.R. China); Marcelina B. Lirazan (Manila, Philippines); Teh-Yung Liu (Taipei, Taiwan); Cheryl E.A. Lovelace (Lusaka, Zambia); Roger L. Lundblad (Duarte, U.S.A.); Marino Martinez-Carrion (Kansas City, U.S.A.); Vincent Massey (Ann Arbor, U.S.A.); John A. McKenzie (Melbourne, Australia); Matthew J. McQueen (Hamilton, Canada); Alan H. Mehler (Washington DC, U.S.A.); Pedro Moradas Ferreira (Porto, Portugal); Yutaka Muto (Tokyo, Japan); Phillip Nagley (Clayton, Australia); Marilou G. Nicolas (Manila, Philippines); Osamu Nureki (Tokyo, Japan); Tatsuo Oka (Isehara City, Japan); Peter Ott (Bern, Switzerland); Vaclav Paces (Prague, Czech Republic); M. Iqbal Parker (Cape Town, South Africa); Armando J. Parodi (Buenos Aires, Argentina); Perumal R. Ramasamy (Kuala Lumpur, Malaysia); Gerry Rank (Saskatoon, Canada); M.R.S. Rao (Bangalore, India); J. Steven Richardson (Saskatoon, Canada); Claudina Rodrigues-Pousada (Oeiras, Portugal); Wilfried Rombauts (Leuven, Belgium); Julian I. Rood (Clayton, Australia); George Russev (Sofia, Bulgaria); Murray Saffran (Toledo, U.S.A.); William H. Sawyer (Melbourne, Australia); Ann Sefton (Sydney, Australia); Giorgio Semenza (Zurich, Switzerland); Adrish Sen (Calcutta, India); Maya Simionescu (Bucharest, Romania); Robert D. Simoni (Stanford, U.S.A.); Willy Stalmans (Leuven, Belgium); Dimitri Stathakos (Athens, Greece); Bruce Stone (Melbourne, Australia); Jisnuson Svasti (Bangkok, Thailand); Yoshihiro Takeda (Ichikawa, Japan); George N. Thomopoulos (Thessaloniki, Greece); Vincent Titanyi (Buea, Cameroon); Kostas Triantaphyllidis (Thessaloniki, Greece); Peter Swann (London, U.K.); Alan C. Taylor (Glasgow, Scotland); H.G. van Eijk (Rotterdam, The Netherlands); Marc H.V. van Regenmortel (Strasbourg, France); Andrea Vasella (Zurich, Switzerland); Frank Vella (Saskatoon, Canada); Jan W. Verhoeven (Amsterdam, The Netherlands); Pirkko Vihko (Oulu, Finland); R. Gerry Wake (Sydney, Australia); Harold B. White III (Newark, U.S.A.); Edward J. Wood (Leeds, U.K.); David Woods (Grahamstown, South Africa)

to the IUBMB Committee on Education for trusting us with this work:

Leopoldo de Meis (Rio de Janeiro, Brazil); Trevor R. Anderson (Scottsville, South Africa); Paolo Bernardi (Padova, Italy); Paul Cohen (Paris, France); Masamitsu Futai (Osaka, Japan); Andre Goffeau (Louvain-la-neuve, Belgium); Cecilia Hidalgo (Santiago, Chile); Giuseppe Inesi (Baltimore, U.S.A.); Charles A. Pasternak (Oxford, U.K.); Giorgio Semenza (Zurich, Switzerland); Edward J. Wood (Leeds, U.K.)

to the IUBMB Executive Committee for encouragement and financial support: 
William J. Whelan (Miami, U.S.A.); Brian F.C. Clark (Aarhus, Denmark); Frank Vella (Saskatoon, Canada); E.C. (Bill) Slater (Lymington, U.K.); William J. Lennarz (New York, U.S.A.); Leopoldo de Meis (Rio de Janeiro, Brazil);

Yasuhiro Anraku (Yamanashi, Japan); Angelo Azzi (Bern, Switzerland); Vito Turk (Ljubljana, Slovenia). 